\title{
CASO
}

\section{ABSCESO DE PSOAS}

\author{
PSOAS ABSCESS \\ Lock Vargas Yatsen ${ }^{1}$, Joo Fernández Hoshe, Díaz Villanueva Patricia², Campana Román \\ Priscilla ${ }^{3}$, Valencia Avalo Daniel $^{4}$
}

\section{RESUMEN}

El absceso de psoas es una enfermedad relativamente rara. Muchos casos presentan características clínicas atípicas. El absceso de psoas puede ser primario en pacientes inmunodeprimidos o secundario a infecciones gastrointestinales y genitourinarias. En los países no desarrollados, la mayoría de estos pueden ser de etiología tuberculosa. Una alta sospecha clínica, una buena historia clínica y estudios de imagen, pueden ser útiles en el diagnóstico y tratamiento de la enfermedad. El tratamiento temprano con antibióticos, drenaje guiado o cirugía abierta es necesario debido a su alta morbimortalidad.

Se reporta el caso de un paciente varón de 57 años con historia de Diabetes mellitus (DM) de 20 años de evolución, quien refiere dolor en región lumbar izquierda asociada a aumento de volumen y fiebre intermitente, por lo que se hospitaliza, detectándose un absceso de psoas izquierdo, el cual es drenado por cirugía abierta con abordaje extraperitoneal, presentando evolución favorable.

\section{ABSTRACT}

Psoas abscess is a relatively rare disease. Many cases present atypical clinical features. Psoas abscess may be primary in immunosuppressed patients or secondary to gastrointestinal and genitourinary infections. In non-developed countries most of these can be of tuberculous etiology. A high clinical suspicion, a good clinical history and imaging studies can be useful in diagnosing the disease. Early treatment with antibiotics, guided drainage or open surgery is necessary due to its high morbidity and mortality.

It's reported the case of a male patient of 57 years with a history of diabetes mellitus (DM) of 20 years of evolution, who referred pain in the left lumbar region associated with increased volume and intermittent fever, so he is hospitalized, detecting an extensive left psoas abscess which is drained by open surgery with extraperitoneal approach, showing favorable evolution.

\section{INTRODUCCIÓN}

$\mathrm{D}$ entro de las patologías quirúrgicas, el absceso de psoas es una condición poco común, de difícil diagnóstico y clínica inespecífica, pero que conlleva complicaciones de muy alta mortalidad, por lo cual su estudio minucioso para el diagnóstico y tratamiento precoz, es fundamental para salvaguardar la vida del paciente, en virtud de ello se plantea el presente reporte de caso en el cual se busca analizar los factores y sucesos que devinieron en la decisión de su drenaje por vía quirúrgica, al no contar con medios menos invasivos en nuestra institución, logrando con ello su resolución sin complicaciones. La alta susceptibilidad del músculo psoas a infecciones está relacionado con su anatomía peculiar; los organismos pueden acceder a este músculo ya sea por extensión directa o por medio de la siembra hematógena'. El músculo psoas se origina desde las vértebras torácicas inferiores hasta las últimas vértebras lumbares (bordes laterales de T12 a L5), y se extiende a través del retroperitoneo hasta insertarse en el trocánter menor del fémur. Por lo tanto, se encuentra en estrecha proximidad a un número de órganos retro e intraabdominales, incluyendo los riñones, uréteres, páncreas, el apéndice, el intestino grueso y delgado ${ }^{2}$. Los organismos relacionados a procesos infecciosos frecuentes de estos órganos podrían extenderse directamente al músculo psoas. Por otra parte, el abundante suministro de sangre al músculo psoas, es considerado un factor predisponente
${ }^{1}$ Médico Asistente del Servicio de Cirugía del Hospital II Lima Norte Callao - Luis Negreiros Vega - ESSALUD.

${ }^{2}$ Médico Asistente del Servicio de Cirugía del Hospital II Gustavo Lanatta Luján -ESSALUD.

${ }^{3}$ Médico Asistente del Servicio de Cirugía del Hospital III Guillermo Almenara Yrigoyen - ESSALUD

${ }^{4}$ Médico Residente de Cirugía del Hospital II Lima Norte CaIlao - Luis Negreiros Vega - ESSALUD

Correspondencia a: Nombre: Yatsen Lock Vargas yalov20@gmail.com

Telf. y celular: 00(51) 1 950074448

Palabras clave: Absceso de psoas, Músculos psoas, Dolor de la región lumbar

Keywords: Psoas abscess, Psoas muscles, Low back pain

Procedencia y arbitraje: no comisionado, sometido a arbitraje externo.

Recibido para publicación 29 de Abril 2018 Aceptado para publicación: 14 de Julio 2018

Citar como: Rev Cient Cienc Med 2018; 20(1):113-117 
para diseminación hematógena desde fuentes distantes². La presentación clínica del absceso de psoas suele ser insidiosa, la triada clínica clásica consiste en la presentación de fiebre, dolor de espalda y masa en región lumbar, pero esta característica solo se observa en la minoría de pacientes ${ }^{3}$. Tradicionalmente el absceso de psoas es clasificado en primario o secundario, dependiendo de la presencia o ausencia de un foco infeccioso identificable en alguna estructura u órgano adyacente 3 . En la actualidad, el principal agente microbiano asociado a abscesos primarios es el patógeno Staphylococcus aureus y en los abscesos secundarios se reportan en los cultivos los gérmenes entéricos ${ }^{4}$. La Diabetes mellitus y el trauma muscular, incluso microscópico, se consideran como factores predisponentes para la formación de abscesos ${ }^{5,6}$. El tratamiento para esta patología consiste de un drenaje adecuado, ya sea por vía percutánea o quirúrgica, junto con la terapia antibiótica inmediata. Las complicaciones graves como sepsis e incluso muerte podrían ser el resultado de retardo en el diagnóstico y el inicio del tratamiento, y la presencia de comorbilidades también se asocia a un mal pronóstico6. La mayoría de abscesos de psoas pueden ser diagnosticados por ecografía, tomografía axial multicorte (TAC) o resonancia magnética (RM), siendo la tomografía contrastada el método más efectivo ${ }^{3}$. El drenaje percutáneo guiado por imágenes es una técnica segura y un tratamiento efectivo principalmente en los abscesos de etiología primaria y tuberculosa. La cirugía solo debe reservarse cuando no se pueda realizar drenaje percutáneo, y en algunos casos de abscesos secundarios ${ }^{7,8}$.

\section{PRESENTACIÓN DEL CASO}

Paciente varón de 57 años quien refiere un tiempo de enfermedad de 2 meses, caracterizado por dolor punzante en la zona lumbar izquierda de moderada intensidad, y sensación de masa en la misma zona, asociado a fiebre intermitente la cual se cuantifica hasta $39^{\circ} \mathrm{C}$ por lo que se automedicó con AINES (naproxeno $550 \mathrm{mg}$ ) por 1 semana sin mejoría clínica, razón por la cual decide acudir al servicio de Emergencia del Hospital II Lima Norte Callao - Luis Negreiros Vega - ESSALUD, donde, luego de la evaluación del equipo médico de guardia, se decide su hospitalización en el servicio de Medicina. Contaba además con antecedente de DM de larga evolución desde hace 20 años, controlado farmacológicamente con Metformina de $850 \mathrm{mg}$ cada 8 horas.

Al momento del ingreso, el paciente se encontraba con facies dolorosa, diaforético, adelgazado, palidez ++/+++, en regular estado de nutrición e hidratación. Al examen físico regional se observaba tumoración flogótica de $7 \times 3$ $\mathrm{cm}$ aproximadamente en la región lumbar izquierda, dolo- rosa a la palpación y de consistencia renitente, dolor a la flexo-extensión del miembro inferior izquierdo. El resto del examen físico general dentro de parámetros normales. Se solicitan exámenes de laboratorio como hemograma completo y glucosa sérica (ver tabla $\mathrm{N}^{\circ} 1$ ). Se solicita tomografía contrastada, la cual informa colección de paredes gruesas que diseca las fibras musculares del psoas izquierdo y que se extiende hacia la pared ipsilateral y fistuliza a los tejidos blandos adyacentes (Ver figura 1), sin comprometer cápsula renal y con realce periférico tras inyección del contraste,

Tabla N¹ Exámenes de laboratorio

\begin{tabular}{|ll|}
\hline Hemoglobina & $8,5 \mathrm{gr} / \mathrm{dl}$ \\
\hline Leucocitos & 7850 células $/ \mathrm{ml}$ \\
\hline Abastonados & $2 \%$ \\
\hline Segmentados & $86 \%$ \\
\hline Glucosa & $157 \mathrm{mg} / \mathrm{dl}$ \\
\hline
\end{tabular}

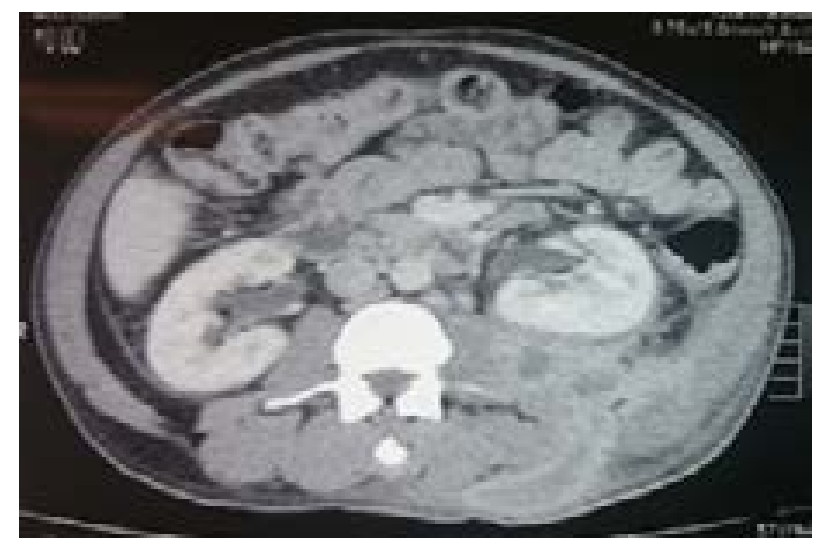

Figura 1. Tomografía con contraste donde se evidencia colección en psoas izquierdo que fistuliza hacia tejido blando.

resto de órganos sin alteraciones. Con los resultados de los exámenes auxiliares se plantea el diagnóstico sugestivo de absceso de psoas izquierdo. Se inicia antibióticoterapia con ciprofloxacino $400 \mathrm{mg}$ EV cada 12 horas más metronidazol $500 \mathrm{mg}$ EV cada 8 horas durante 10 días, y ante la persistencia de picos febriles e imposibilidad de drenaje por radiología intervencionista debido a falta de materiales, se realiza interconsulta al Servicio de Cirugía, decidiéndose un drenaje quirúrgico abierto del absceso. En sala de operaciones, bajo anestesia general, se coloca al paciente en decúbito lateral derecho y se realiza un abordaje extraperitoneal del absceso de psoas izquierdo (Ver figura 2), en los hallazgos se identifica un trayecto fistuloso del tercio inferior del psoas hacia pared lumbar izquierda y colección seropurulenta proveniente del mismo, en volumen aproximado de $100 \mathrm{cc}$. 


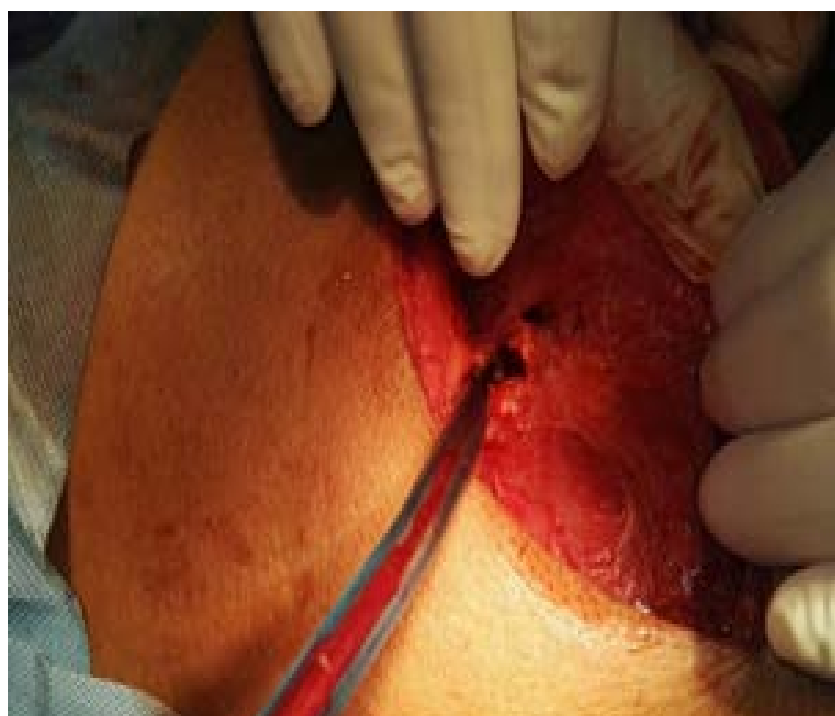

Figura 2. Abordaje extraperitoneal de psoas izquierdo y drenaje de absceso

Se realiza ecografía intraoperatoria para descartar colecciones residuales, en el que observamos cavidad hipoecogénica de $8 \mathrm{~cm}$ de diámetro en tercio inferior de psoas izquierdo. Se lava y coloca un tubo de anodex para drenaje (Ver figura 3). Se envían muestras para cultivos, tinción de GRAM y descarte de tuberculosis. El paciente pasa a Servicio de Medicina continuando antibioticoterapia y ampliación de estudios para descarte de secundarismo. Los picos febriles ceden totalmente y el drenaje seropurulento disminuyó de 35 a 10 cc, por lo que fue dado de alta al séptimo día post operatorio sin el tubo de drenaje y con antibióticoterapia por vía oral hasta completar 2 semanas.

Se descarta secundarismo con resultados negativos, y cultivo positivo para Staphylococcus aureus Meticilino Sensible. El diagnóstico definitivo fue Absceso de Psoas Primario.

\section{DISCUSIÓN}

El absceso de psoas es una condición poco común, en el presente caso tiende a ser de etiología no tuberculosa. El absceso de psoas es un tipo de miositis infecciosa y puede ser causada por una amplia gama de bacterias, hongos, parásitos y agentes virales ${ }^{9}$. Sólo unos pocos casos de abscesos de psoas son reportados por año, siendo primario o secundario a enfermedades gastrointestinales o del tracto genitourinario, inflamatorias, infecciones óseas, en particular de la columna vertebral, y después de una cirugía abdominal ${ }^{10}$. La ausencia de las causas antes mencionadas caracteriza al absceso como primario, donde el tratamiento antibiótico asociado a drenaje guiado por TAC es efectivo, aunque la tasa de recurrencia puede ser más alto que con drenaje quirúrgico ${ }^{11}$. Los abscesos primarios de psoas constituyen el $30-60 \%$ del total de los abscesos de psoas, el $90 \%$

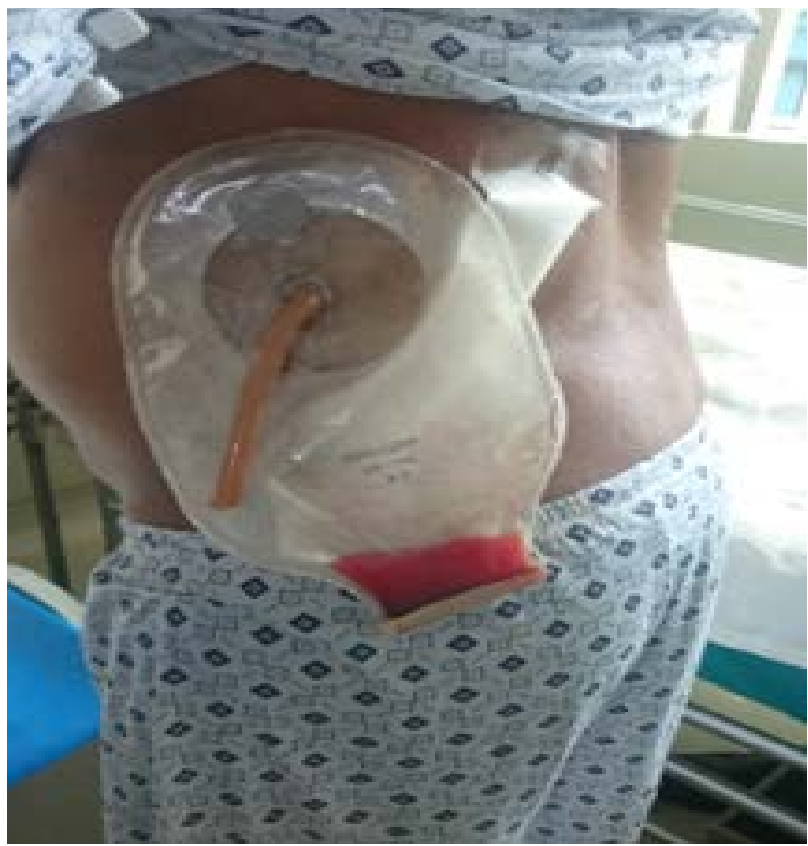

Figura 3. Colocación de tubo de Anodex

de los pacientes tiene menos de 30 años y el $75 \%$ del total de enfermos son varones. ${ }^{4}$ En el pasado, la espondilitis tuberculosa con cambios óseos era una causa frecuente y el proceso inflamatorio podía extenderse a través del músculo psoas iliaco hacia la región inguinal. En la actualidad, el principal agente microbiano en abscesos primarios parece ser el Staphylococcus aureus. Otros agentes microbianos, como Escherichia coli, Pseudomonas aeruginosa y Streptococcus pneumoniae, a veces pueden desempeñar un papel en la formación del absceso. El sitio de entrada del microorganismo por lo general no es evidente, y se extiende por vía hematógena desde un sitio lejano ${ }^{7}$. Los abscesos primarios se presentan por lo general en jóvenes; suelen ser unilaterales y se encuentran con más frecuencia en los países en desarrollo de Asia y África, asociado al aumento en la incidencia de individuos con $\mathrm{VIH}^{12}$. En nuestro caso difiere la edad de presentación de esta patología con lo descrito en la literatura, probablemente se atribuye a la inmunodepresión crónica ocasionada por la DM tipo 2 mal controlada por el paciente. El absceso de psoas secundario esta ocasionado por flora entérica positiva, enfermedades inflamatorias gastrointestinales como apendicitis, enfermedad de Crohn, diverticulitis y el carcinoma de colon son las causas más comunes, mientras que en algunos países de Asia es la patología renal un factor importante para el desarrollo de abscesos ${ }^{13 .}$ Causas poco frecuentes son leucemia crónica, pancreatitis, artritis séptica y la púrpura de Henoch-Schönlein. La mayoría de los abscesos se encuentran en el área derecha del psoas; no tienen predominancia por grupo de edad y son altamente prevalentes en Europa y los países de América ${ }^{8}$. El diagnóstico clínico de un absceso de psoas puede ser difícil y los síntomas clásicos de dolor en el flanco, fiebre y aumento de volumen suelen 
estar ausentes. La clínica de nuestro paciente fue la triada clásica descrita en la literatura, lo cual ayudó a la sospecha del cuadro. El examen de laboratorio frecuentemente muestra anemia y leucocitosis. El diagnóstico precoz puede ser obstaculizado por síntomas clínicos atípicos y el retraso de terapia resulta en aumento de la morbimortalidad. El aparente aumento de la incidencia de abscesos de psoas iliaco se debe al aumento del número de pacientes inmunocomprometidos, la quimioterapia, la inmunoterapia, enfermedad multisistémica y la amplia disponibilidad de imágenes tomográficas ${ }^{14}$. La mayoría de abscesos de psoas se pueden diagnosticar por los estudios de imagen de ecografía, TAC o $\mathrm{RM}$, pero cuando se interpretan las imágenes sin el conocimiento de la historia clínica, el diagnóstico diferencial tales como abscesos, neoplasias y hematomas, puede ser difícil. La TAC es más precisa y puede diagnosticar correctamente la mayoría de los $\operatorname{casos}^{15}$. Ante la sospecha de esta patología en nuestro paciente, se solicitó una TAC abdominal con contraste con la que se logró confirmar el diagnóstico. La RM es útil para la evaluación temprana o en tumores infiltrantes y es muy sensible en la detección de tumor o propagación de la infección en las vértebras adyacentes, discos o canales. El drenaje percutáneo guiado por TAC es una técnica segura y un tratamiento efectivo principalmente en los abscesos de etiología primaria y tuberculosa. La cirugía debe reservarse cuando no se pueda realizar drenaje percutáneo, y en algunos casos de abscesos secundarios. El abordaje quirúrgico para el absceso puede ser realizado por una incisión extraperitoneal cuando el absceso es primario o a través de una incisión abdominal cuando el absceso es secundario a una infección gastrointestinal o tumoración ${ }^{7}$. Lamentablemente no contamos con el Servicio de Radiología Intervencionista a tiempo completo por problemas logísticos, por lo que fue necesario el drenaje a través de un abordaje extraperitoneal abierto, el cual creemos es mejor que el transperitoneal, ya que evitamos la contaminación de la cavidad abdominal, y además se expone de forma amplia y correcta la zona a drenar, ayudándonos también de la ecografía intraoperatoria para asegurarnos de lograr un drenaje completo. El paciente fue catalogado al egreso como un absceso de psoas primario, ya que los exámenes complementarios no mostraron indicios de secundarismo.

\section{CONCLUSIÓN}

La etiología de absceso del psoas puede variar. La presentación clínica es a menudo vaga: el dolor lumbar, hallazgos laboratoriales de sepsis y una buena historia clínica son la base para la sospecha de absceso de psoas. Pacientes inmunocomprometidos, o la presencia de factores de riesgo como la diabetes mellitus y trauma en la zona del psoas, representan a los principales grupos de riesgo. El absceso de psoas puede diagnosticarse fácil y tempranamente con TAC o cualquier otro estudio de imágenes. Es necesario definir un absceso como primario o secundario tan pronto como sea posible para darle el tratamiento más apropiado antes que la septicemia se vuelva letal. El drenaje guiado percutáneo confiere una baja morbilidad y es el tratamiento principal, junto con los antibióticos apropiados, principalmente en abscesos primarios, aunque tiene una mayor tasa de recurrencia en comparación con la cirugía. Cuando falla o si hay una contraindicación, el tratamiento quirúrgico debe llevarse a cabo. En éste último caso la incisión más utilizada para el enfoque del absceso es a través de la vía extraperitoneal, los abscesos secundarios a enfermedades intestinales inflamatorias o carcinomas deben ser tratados mediante cirugía con una incisión abdominal media. La posición de la laparoscopía en el tratamiento de los abscesos de psoas no es muy clara en la actualidad, debido a la rareza de la enfermedad y las pocas referencias en la literatura ${ }^{16}$. El papel de los antibióticos es importante para el tratamiento final de los abscesos del psoas.

\section{REFERENCIAS}

1.Crum-Cianflone NF. Bacterial, fungal, parasitic, and viral myositis. Clin Microbiol Rev [Internet]. 2008 [Acceso 8 de noviembre 2016];(21):473-94. Disponible en: https://www.ncbi.nlm.nih.gov/ pmc/articles/PMC2493084/

2.Mallick IH, Thoufeeq MH, Rajendran TP. Iliopsoas abscesses. Postgrad Med J. [Internet].2004 Aug [Acceso 15 de noviembre 2016];80(946):459-62. Disponible en: https://www.ncbi.nlm.nih. gov/pmc/articles/PMC1743075/pdf/v080p00459.pdf

3.Cánovas JA, Tramoyeres A, Sánchez F, Ramos M, et al. Absceso de psoas primario: a propósito de 5 nuevos casos. Revisión de la literatura. Arch. Esp. Urol. [Internet]. 2003 [Acceso 21 de noviembre 2016];(56):775-80. Disponible en: http://aeurologia.com/pdfs/ articulos/5215921048530-eng.pdf

4. Yacoub WN, Sohn HJ, Chan S, Petrosyan M, Vermaire HM, Kelso $\mathrm{RL}$, et al. Psoas abscess rarely requires surgical intervention. Am J Surg [Internet]. 2008 [Acceso 21 de noviembre 2016];(196):22327. Disponible en: http://www.americanjournalofsurgery.com/article/S0002-9610(08)00181-5/fulltext
5.Garner JP, Meiring PD, Ravi K, Gupta R. Psoas abscess: not as rare as we think? Colorectal Dis [Internet]. 2007 [Acceso 8 de noviembre 2016];(9):269-74. Disponible en: http://onlinelibrary.wiley. com/doi/10.1111/j.1463-1318.2006.01135.x/pdf

6. Wells RD, Bebarta VS. Primary iliopsoas abscess caused by community-acquired methicillin-resistant Staphylococcus aureus. Am J Emerg Med [Internet]. 2006 [Acceso 14 de noviembre 2016];(24):897-98. Disponible en: http://www.ajemjournal.com/article/S0735-6757(06)00139-2/fulltext

7.Huang JJ, Ruaan MK, Lan RR, Wang MC. Acute pyogenic iliopsoas abscess in Taiwan: clinical features, diagnosis, treatments and outcome. J Infect Dis [Internet].2000 [Acceso 22 de noviembre 2016];(40):248-55. Disponible en: http://www.sciencedirect.com/ science/article/pii/S0163445300906431?via\%3Dihub

8.Van den Berge M, De Marie S, Kuipers T, Jansz AR, Bravenboer B. Psoas abscess: report of a series and review of the literature. Neth J Med [Internet]. 2005 [Acceso 14 de noviembre 2016];(63):13-16. Disponible en: http://www.njmonline.nl/getpdf.php?id=367 
9.Navarro V, López F, González E, Gregori J, Munoz A. Psoas abscess in patients infected with the human immunodeficiency virus. Clin Microbiol Infect Dis [Internet]. 2004 [Acceso 15 de noviembre 2016];(23):661-63. Disponible en: https://link.springer. com/article/10.1007\%2Fs10096-004-1173-x

10.García P, Laguna P, López-Cano M, Castañeda A, Gil M. Abscesos piógenos y tuberculosos del músculo psoas. Rev Clin Esp [Internet]. 2011 Dec [Acceso 28 de noviembre 2016];211(11):57278. Disponible en: https://www.clinicalkey.es/service/content/pdf/ watermarked/1-s2.0-S0014256511004139.pdf?locale=es_ES

11.ShieldsDW, RobinsonP, CrowleyTP.Iliopsoas abscess--a review and update on the literature. Int J Surg [Internet]. 2012 [Acceso 28 denoviembre2016];10(9):466-69. Disponible en: https://www.clinicalkey.es/\#!/content/playContent/1-s2.0-S1743919112007650?returnurl=http: $\% 2 F \% 2$ Flinkinghub.elsevier.com $\% 2$ Fretrieve $\%$ 2 Fpii\%2FS1743919112007650\%3Fshowall\%3Dtrue\&referrer $=$ https: $\% 2 F \% 2 F w w w . n c b i . n l m . n i h . g o v \% 2 F$

12.Cronin CG, Lohan DG, Meehan CP, Delappe E, McLoughlin R, O'Sullivan GJ, et al. Anatomy, pathology, imaging and intervention of the iliopsoas muscle revisited. Emerg Radiol [Internet]. 2008 [Acceso 8 de noviembre 2016];(15):295-310. Disponible en: https://link.springer.com/article/10.1007\%2Fs10140-008-0703-8

13.Cantasdemir M, Kara B, Cebi D, Selcuk ND, Numan F. Computed tomography-guided percutaneous catheter drainage of primary and secondary iliopsoas abscesses. Clin Radiol [Internet]. 2003 [Acceso 14 de noviembre 2016];(58):811-15. Disponible en: http://www.clinicalradiologyonline.net/article/S0009-

\section{0(03)00274-5/fulltext}

14.Fernández-Ruiz M, Estébanez-Muñoz M, López-Medrano F, Aguado JM. Absceso del músculo iliopsoas: tratamiento y evolución en una serie de 35 pacientes. Enferm Infecc Microbiol Clin [Internet].2012 Jun [Acceso 7 de noviembre 2016];30(6):30711. Disponible en: https://www.clinicalkey.es/service/content/pdf/ watermarked/1-s2.0-S0213005X11003181.pdf?locale $=e s \_E S$

15.Obata A, Kaneto H, Kamei S, Shimoda M, Kimura T, Hirukawa $\mathrm{H}$, et al. Case of iliopsoas abscess that was markedly recovered after percutaneous and surgical drainage in a patient with poorly controlled type 2 diabetes. J Diabetes Investig [Internet]. 2016 May [Acceso 7 de noviembre 2016];7(3):440-41. Disponible en: https://www.ncbi.nlm.nih.gov/pmc/articles/PMC4847901/

16.Kao PF, Tsui KH, Leu HS, Tsai MF, Tzen KY. Diagnosis and treatment of pyogenic psoas abscess in diabetic patients: usefulness of computed tomography and Gallium-67 scanning. Urology [Internet]. 2001 [Acceso 21 de noviembre 2016];(57):246-51. Disponible en: http://www.sciencedirect.com/ science/article/pii/S0090429500009237 